\title{
Chronic Total Coronary Artery Occlusion Recanalization with Percutaneous Coronary Intervention Using Anterograde and Retrograde Approach - Riga East Clinical University Hospital Experience
}

\author{
Artis Kalnins*, Guntis Lukstins*, Irena Kurcalte *, Aivars Lejnieks ** \\ * Riga EAST University hospital, Riga, Latvia \\ ** Riga Stradins University, Riga, Latvia
}

\begin{abstract}
Summary
Introduction. Despite advantages in interventional cardiology during last decade, chronic total occlusions (CTO) still remains one of the biggest problem in percutaneous coronary interventions (PCI) $(10,14)$. CT0 prevalence is high, but only less than $10 \%$ of percutaneous revascularizations are CTO interventions (17). There are no prospective randomized trials, properly powered for hard clinical endpoints, comparing modern optimal medical therapy with contemporary state of the art CT0 recanalization (6).

Aim of the study. The aim of our study was to compare CTO PCI procedural parameters and treatment results using anterograde and/or retrograde approach.

Materials and methods. The study included all patients undergoing PCI for CTO at single tertiary PCI center between January 2007 and December 2012. $5568 \mathrm{PCl}$ procedures were done in this period in our institution. $486(8,64 \%)$ of them were CTO PCl. Retrograde approach was used in 138 ( $28,7 \%$ of all CTO PCI) cases. Patients were grouped according PCl year performing, approach (anterograde or retrograde) and PCI results (successful or unsuccessful). Demographic and procedural data were collected at the time of intervention.

Results. A total of 405 patients undergoing CTO PCI were included. The median age was $64 y$ rs(38-88) and $79,2 \%$ was male. Retrograde approach (RA) was used in $138(28,7 \%$ ) cases. RA usage has increase from $15.9 \%$ in 2007 till $46,8 \%$ of cases in $2012(p=0,0000218)$. The overall patient and procedure success rates were $77,8 \%(315 / 90)$ and $69,9 \%(340 / 146)$ respectively. Overall success rate has increase from $61,4 \%$ in 2007 till $87,1 \%$ in $2012(p<0,001)$.

Overall survival was found better in patients group after successful procedure (Long-rank test $\mathrm{p}=0,019$ ).

Conclusions. Retrograde approach usage significantly increase CTO PCI success rate, but doesn't increase risk of complications. Long-term outcome and survival after CTO PCI is not depending on approach (anterograde or retrograde), but on procedural success.
\end{abstract}

Key words: chronic total coronary artery occlusion; percutaneous coronary intervention; anterograde and retrograde approach.

\section{INTRODUCTION}

Despite advantages in interventional cardiology during last decade, chronic total occlusions (CTO) still remains one of the biggest problem in percutaneous coronary interventions $(\mathrm{PCI})(10,14)$. According data from registries, CTO prevalence is high - CTO are encountered in $15 \%$ to $30 \%$ of patients undergoing coronary angiography (17). Compared with failed CTO PCI, successful CTO opening has been associated with angina relief, improvement of left ventricular function, avoidance of coronary artery bypass grafting and increased survival. Despite these benefits, less than $10 \%$ of percutaneous revascularizations are CTO interventions (17). CTO PCI is performed infrequently (4), likely due to historically low procedural success rates, technical complexity, high equipment use and the potential for major procedural complications (13).
PCI of CTO are also still relatively infrequent due to the uncertainty and paucity of data on the likelihood of successful PCI of CTO. There are no prospective randomized trials, properly powered for hard clinical endpoints, comparing contemporary optimal medical therapy with contemporary state of the art CTO recanalization (6).

The anterograde approach is the first technique chosen in the majority of PCI CTOs. However in many cases, antegrade wiring fails to cross CTO lesions.

In 1990, the first report of retrograde approach for СТO was published, in which the retrograde wire crossing technique was applied via a degenerated saphenous vein graft (SVG)(8). Later septal collaterals are considered to be potential access for retrograde approach. In 2005 Katoh and colleagues opened a new era of retrograde CTO recanalization with the Controlled Anterograde 
and Retrograde sub intimal Tracking (CART) technique (15). Different kinds of retrograde techniques were introduced in the last years. Novel techniques hold promise in the field of percutaneous coronary intervention (PCI) for CTO (12).

\section{AIM OF THE STUDY}

The aim of our study was to compare CTO PCI procedural parameters and treatment results using anterograde and/or retrograde approach.

\section{MATERIALS AND METHODS}

Definition: A CTO lesion was defined as an obstruction of a coronary artery with Thrombolysis in Myocardial Infarction (TIMI) flow grade 0 with an estimated duration of at least 3 months. The duration of the occlusion was determined by the interval from the last episode of acute coronary syndrome consistent with the location of the occlusion or proven by previous angiography (2).

Multicenter CTO Registry in Japan has established as scoring system known as J-CTO score which can grade the difficulty in crossing a chronic total occlusion (CTO) of a native coronary lesion. The score was derived from the analysis of nearly five hundred native CTO lesions. J CTO score was defined as CTO complexity ratio, which is sum of anatomical findings and previous attempt results - predictors of procedure failure: blunt stump, CTO length $>20 \mathrm{~mm}$, presence of severe calcification, proximal segment tortuosity and previous attempt failure (9).

The study included all patients undergoing PCI for CTO at single tertiary PCI center between January 2007 and December 2012. 5568 PCI procedures were done in this period in our institution. $486(8,64 \%)$ of them were CTO PCI. Retrograde approach was used in $138(28,7 \%$ of all CTO PCI) cases. Patients were grouped according PCI year performing, approach (anterograde or retrograde) and PCI results (successful or unsuccessful). Demographic and procedural data were collected at the time of intervention (Table 1, 2). In-hospital MACE (myocardial infarction, urgent revascularization, stroke or death) was documented at discharge. Post discharge data was obtained by telephone follow up.

Descriptive analysis was used. Continuous variables were presented as mean \pm standard deviation (SD). Categorical variables were presented as incidence (\%). Statistical analysis was performed using SPSS 15.0 statistical software package.

\section{RESULTS}

A total of 405 patients undergoing PCI for 486 CTO were included. Number of patients and number of procedures was different, because 9 patients had 2 vessels CTO, but for 60 patients CTOs was attempted open twice or three times. The median age was $64 y$ rs(38-88) and $79,2 \%$ was male. Retrograde approach (RA) was used in $138(28,7 \%)$ cases. RA usage has increase from $15.9 \%$ in 2007 till $46,8 \%$ of cases in 2012 ( $p=0,0000218$ ). (Figure 5) The overall patient and procedure success rates were
$77,8 \%(315 / 90)$ and 69,9\%(340/146) respectively. Overall success rate has increase from $61,4 \%$ in 2007 till $87,1 \%$ in $2012(p<0,001)$ (Figure 4).

The target artery with CTO lesions included the left main ( 3 cases, $0,62 \%)$ right coronary artery $(270$ cases, 56,1\%), the left anterior descending artery (165 cases, $34,3 \%$ ), and the left circumflex artery (42 cases, $8,73 \%)$. Retrograde approach usage was for RCA 34\%, LAD $23 \%$, LCX $17 \%$ of cases.

We didn't find significant differences in patients clinical characteristics undergoing anterograde and retrograde procedures, except prior Coronary Artery Bypass Grafting $(\mathrm{CABG})$. In retrograde approach patients group prior CABG had $15,2 \%$, in anterograde group $6,4 \%$ patients. Mean CTO duration was longer in retrograde group - 50,4 month versus 35,4 month in anterograde group (Table 1).

Patients with diabetes (insulin dependent and non insulin dependent) was $22,45 \%$ in anterograde approach group and $17,5 \%$ in retrograde approach group $(p=N S)$ (Table 1).

Three strategies for retrograde approach were applied: retrograde as primary strategy, retrograde immediately after antegrade failure and repeat procedure after previous antegrade failure. Retrograde approach as the primary strategy was applied in $29 / 137$ (21,17\%) patients, retrograde approach immediately after antegrade failure attempt was performed in 99/137 $(72,26 \%)$ patients, and retrograde approach as elective procedure, after previously failed antegrade attempt, was performed in $9 / 137(6,57 \%)$ patients. The success rate of these strategies was: 55\% (20/23 patients) for primary, $61 \%$ (7/8 patients) for retrograde immediately after antegrade failure, and 44\% (8/9 patients) for retrograde after previous failed antegrade attempt, respectively. Better success rate $(61 \%)$ were founded in procedures group, whitch was done in one session - retrograde approach immediately after failed anterograde in comparison with 55\% and $44 \%$ success rates in retrograde as primary and retrograde after previous failed anterograde respectively (Figure 6).

J CTO score was calculated for all patients. For less complex CTO lesions( J CTO score 0-1) retrograde approach was used in $5,1 \%$ and $19,8 \%$ of cases respectively, for intermediate complex lesions ( $\mathrm{J}$ CTO score 2 and 3 ) in 28,5\% and $42,3 \%$ respectively, for very complex lesions ( $\mathrm{J}$ CTO score 4 ) in $70,6 \%$ of cases $(\mathrm{p}<0,001)$.

Septal collaterals were more frequently used as the retrograde access route $(92.9 \%)$, but during last two years epicardial channels usage has increse.

The main reasons for failure in both groups (anterograde and retrograde) was inability to cross the occlusion with a wire $(68,7 \%$ of all unsuccessful cases) (Table 2$)$.

Total complications rate was $13,7 \%$.In anterograde patients group $11,4 \%$, in retrograde $19,6 \%(p=0,018)$. Main complications were coronary artery perforation 6 cases in anterograde group (1,75\% of all anterograde cases), 1 in retrograde $(0,73 \%$ of all RA cases $)$; intramural hemoatoma - 5 cases in anterograde group $(1,46 \%$ of 
all anterograde cases), 5 in retrograde $(3,64 \%$ of all RA cases), access site complications -5 cases in anterograde group (1,46\% of all anterograde cases), 3 in retrograde $(2,19 \%$ of all RA cases) (Table 3$)$.

In anterograde procedures group was found better survival results in successful procedures group, but difference was not significant (Long-rank test $p=0,192$ ). In retrograde procedures group also better survival was found in successful procedures group and difference was significant (Long-rank test $\mathrm{p}=0,012$ ) (Figure 2). In unsuccessful procedures group was found similar survival results in both - anterograde and retrograde groups (Long-rank test $\mathrm{p}=0,751$ ).

Overall survival was found better in patients group after successful procedure (Long-rank test $\mathrm{p}=0,019$ ) (Figure $1-3)$.

\section{DISCUSSION}

Revascularization of CTO, similar to stenotic vessels, is indicated in the presence of angina or ischemia related to the respective territory. The clinical presentation of a CTO can be very variable. On the one hand, there are patient with stable angina, silent ischemia or heart failure of ischemic origin. On the other hand for some patients CTO is incidental finding. Potential benefits of occluded artery opening are angina relief, left ventricular function improvement, coronary artery bypass avoiding (11).

Most of CTO patients are less symptomatic to compare with patients with stenotic lesions. Multiple registry experiences suggest that many patients with CTO do not receive either surgical bypass or percutaneous revascularization for the involved artery territory. Also multiple registers shows data suggest benefit of CTO opening $(1,7,16)$. However not all CTOs benefit from revascularization. Main question is do we improve the prognosis with CTO revascularization. In Occluded Artery Trial (OAT) patients with a recent myocardial infarction of 3-28days, the interventional approach showed no advantage in terms of survival and more recurrent myocardial infarctions than in conservative approach. However this trial dealing with a different subset of patients, who had infarctions and only poor proof of viability or ischemia (5). In the Euro CTO register, the biggest ongoing CTO registry, only $18 \%$ of the patients presented with $\mathrm{Q}$ waves in the territory of the CTO vessel (11).

The main finding of this study is that the retrograde approach can be an effective tool for increasing the success rate of recanalization in complex CTO. Also study shows better survival after successful CTO opening. First successful CTO PCI using retrograde approach in Riga EAST hospital was done in December 2006. During last years operators have improve skills and retrograde approach usage in CTO cases have increased till $50 \%$. Retrograde CTO cases was more complex, average J CTO score was 1,54 in anterograde and 2,45 in retrograde procedures group and better overall survival has been reached owing better results in retrograde group.

Retrograde approach is associated with potentially higher risk of complications. In our study we found higher complications rete in retrograde patients group, but difference was not statistically significant.

\section{CONCLUSIONS}

Retrograde approach usage significantly increase CTO PCI success rate. More complex technique (retrograde approach) usage doesn't significantly increase rate of complications. Indication of retrograde approach should be performed in case anterograde wiring seems very difficult in terms of anatomical factors. Long-term outcome and survival after CTO PCI is not depending on approach (anterograde or retrograde), but on procedural success.

\section{Conflict of interest: None}

\section{REFERENCES}

1. Aziz S, Stables RH, Grayson AD, et al. Percutaneous coronary intervention for chronic total occlusions: improved survival for patients with successful revascularization compared to a failed procedure // Catheter Cardiovasc Interv 2007; 70:15-20

2. Ge L, Iakovou I, Cosgrave J, Chieffo A, Montorfano M, Michev I, et al. Immediate and mid-term outcomes of sirolimus-eluting stent implantation for chronic total occlusions // Eur Heart J 2005; 26:1056-1062

3. Ge Lei, Qian Ju-ying, Liu Xue-bo, et.al. Retrograde approach for the recanalization of coronary chronic total occlusion: preliminary experience of a single center // Chinese Medical Journal 2010; 123(7):857-863

4. Grantham JA, Marso SP, Spertus J, et al. Chronic total occlusion angioplasty in the United States //.J.Am Coll Cardiol Interv 2009; 2:479-86

5. Hochman JS, Lamas GA, Buller CE, et al. Coronary intervention for persistent occlusion after myocardial infarction // $\mathrm{N}$ Engl J Med, 2006;355(23):2395-407

6. Hoye A, van Domburg RT, Sonnenschein K, et al. Percutaneous coronary intervention for chronic total occlusions: the Thoraxcenter experience 1992-2002 //Eur Heart J 2005; 26:2630-6

7. Joyal D, Afilalo J, Rinfert S. Effectiveness of recanalization of chronic total occlusions: a systematic review and meta-analysis //Am Heart J, 2010;160(1):179-87

8. Kahn JK, Hartzler GO. Retrograde coronary angioplasty of isolated arterial segments through saphenous vein bypass grafts //Cathet Cardiovasc Diagn 1990; 20: 88-93

9. Morino $\mathrm{Y}$, Abe $\mathrm{M}$, Morimoto $\mathrm{T}$, Kimura $\mathrm{T}$, et al. Predicting successful guide wire crossing through chronic total occlusion of native coronary lesions within 30 minutes: the J-CTO (Multicenter CTO Registry in Japan) score as a difficulty grading and time assessment tool // JACC Cardiovasc Interv. 2011 Feb;4(2): 213-21 
10. Prasad A, Rihal CS, Lennon RJ, Wiste HJ, Singh M, Holmes DR. Trends in outcomes after percutaneous coronary intervention for chronic total occlusions: a 25year experience from the Mayo Clinic // J Am Coll Cardiol 2007; 49: 1611-1618

11. Reifart N. Percutaneous Revascularization of Coronary Chronic Total Occlusion-Outcomes and Development of Strategy 2006-2010 // European Cardiology, 2011 ; 7(4):288-293

12. Saito S. Different strategies of retrograde approach in coronary angioplasty for chronic total occlusion // Catheter Cardiovasc Interv 2008; 71:8-19

13. Shah PB. Management of coronary chronic total occlusion. Circulation 2011; 123:1780-4

14. Suero JA, Marso SP, Jones PG, Laster SB, Huber KC, GiorgiLV, et al. Procedural outcomes and long-term survival among patients undergoing percutaneous coronary intervention of a chronic total occlusion in native coronary arteries: a 20-year experience // J Am Coll Cardiol, 2001; 38: 409-414

15. Surmely JF, Tsuchikane E, Katoh O, et al. New concept for CTO recanalization using controlled anterograde and retrograde subintimal tracking: the CART technique // J Invasive cardiol, 2006;18(7):334-338

16. Valenti R, Migliorini A, Signorini U, et al. Impact of complete revascularization with percutaneous coronary intervention on survival in patients with at least one chronic total occlusion // Eur Heart J, 2008;29(19):2336-42

17. Werner GS, Gitt AK, Zeymen U et al. Chronic total coronary occlusions in patients with stable angina pectoris: impact on therapy and outcome in present ay clinical practice // Clin Res Cardiol, 2009, 98(7); 435-41

\section{Address:}

Artis Kalnins

Clinic of Heart vessel diseases

Riga EAST University hospital

Riga, LV 1038, Latvia

e-mail: artis.kalnins@aslimnica.lv

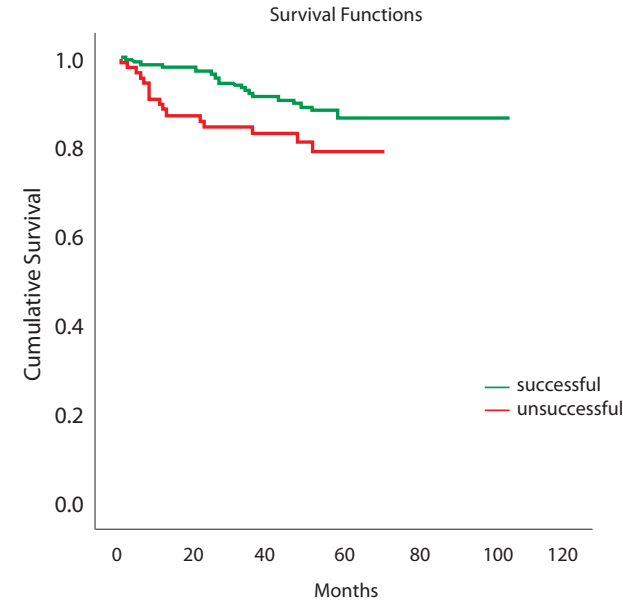

Fig. 1. Survival. Successful and unsuccessful cases

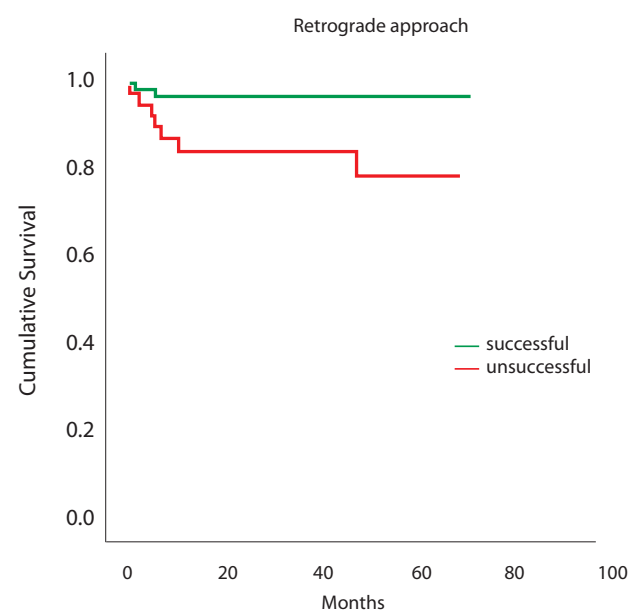

Fig. 2. Survival. Retrograde approach

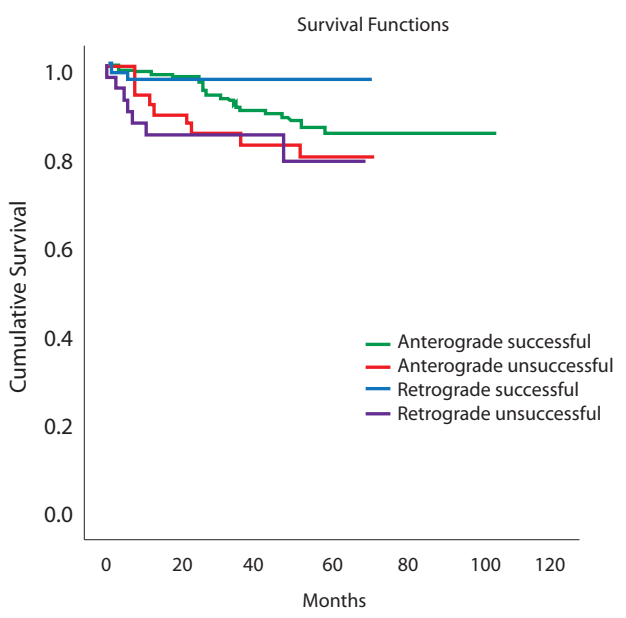

Fig. 3. Overall survival retrograde/ anterograde approach, successful/unsuccessful cases 


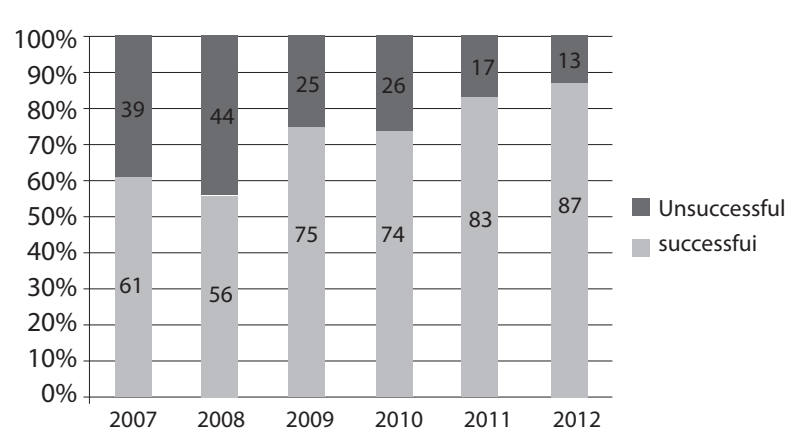

Fig. 4. Overall CTO PCI success rate in Riga EAST hospital

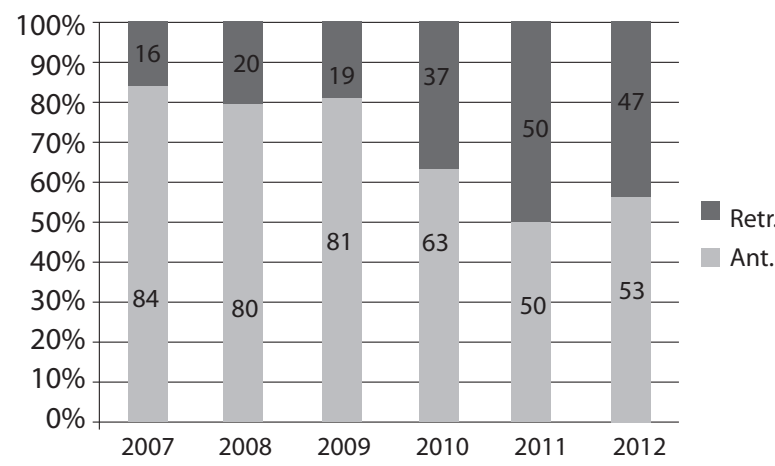

Fig. 5. Anterograde and retrograde approach usage in CTO cases

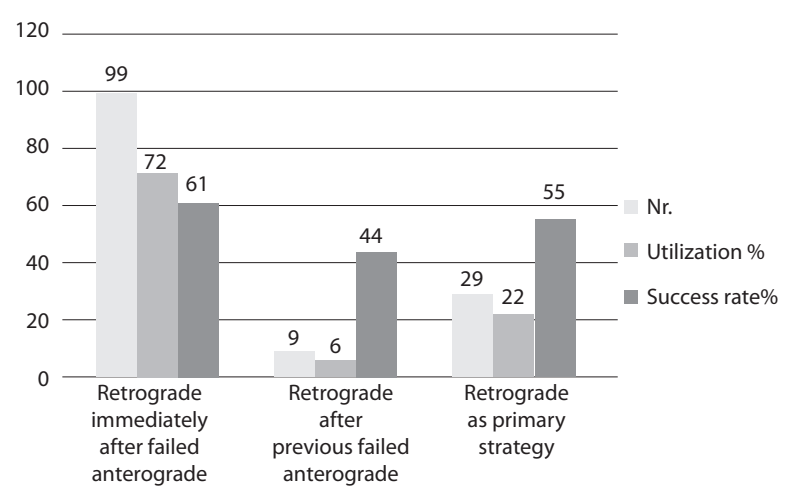

Fig. 6. CTO Retrograde cases strategy and success $\operatorname{rates}(n=137)$
Table 1.CTO PCI patients baseline clinical characteristics $(\mathrm{N}=\mathbf{4 0 5})$

\begin{tabular}{|c|c|c|c|}
\hline Variable & Anterograde & Retrograde & $\mathrm{p}$ \\
\hline Age & $\begin{array}{c}63,11(+/- \\
10,28)\end{array}$ & $\begin{array}{c}63,49(+/- \\
9,99)\end{array}$ & NS \\
\hline Male & $78,4 \%$ & $81,15 \%$ & NS \\
\hline Diabetes & $22,45 \%$ & $17,5 \%$ & NS \\
\hline Smokers & $32 \%$ & $34 \%$ & NS \\
\hline Hypertension & $55 \%$ & $61 \%$ & NS \\
\hline Hyperlipidemia & $47 \%$ & $44 \%$ & NS \\
\hline Prior MI & $53,3 \%$ & $50,0 \%$ & NS \\
\hline Prior CABG & $6,4 \%$ & $15,2 \%$ & $<0,001$ \\
\hline $\begin{array}{c}\text { Mean CTO } \\
\text { duration } \\
\text { (month) }\end{array}$ & 35,4 & 50,4 & $<0,001$ \\
\hline
\end{tabular}

Table 2. Reasons for CTO procedure failure

\begin{tabular}{|c|c|c|}
\hline & $\begin{array}{c}\text { Anterograde } \\
\text { failure } \\
\mathrm{N}=89\end{array}$ & $\begin{array}{c}\text { Retrograde } \\
\text { failure } \\
\mathrm{N}=58\end{array}$ \\
\hline $\begin{array}{c}\text { Wire was unable } \\
\text { cross collaterals }\end{array}$ & - & 14 \\
\hline $\begin{array}{c}\text { Ballon delivery } \\
\text { failure }\end{array}$ & 8 & 4 \\
\hline $\begin{array}{c}\text { Wire was unable to } \\
\text { get through CTO } \\
\text { body }\end{array}$ & 75 & 25 \\
\hline Complications & 6 & 2 \\
\hline $\begin{array}{c}\text { Hight contrast } \\
\text { volume, radiation } \\
\text { explosure }\end{array}$ & 0 & \\
\hline
\end{tabular}

